\title{
Identification of protein interaction antagonists using the repressed transactivator two-hybrid system
}

\author{
Phalgun B. Joshi¹, Martin Hirst1, Tom Malcolm², Jennifer Parent², David Mitchell1, Karen Lund¹, \\ and Ivan Sadowski1,2
}

BioTechniques 42:635-644 (May 2007)

doi 10.2144/000112434

\begin{abstract}
The repressed transactivator (RTA) yeast two-hybrid system was developed to enable genetic identification of interactions with transcriptional activator proteins. We have devised modifications of this system that enable its use in screening for inhibitors of protein interactions from small molecule compound libraries. We show that inhibition of protein interactions can be measured by monitoring growth in selective medium containing 3-aminotriazole (3-AT) and using this assay have identified inhibitors of four independent protein interactions in screens with a 23,000 small molecule compound library. Compounds found to inhibit one of the tested interactions between FKBP12 and the transforming growth factor $\beta$ receptor (TGF $\beta$ - $R$ ) were validated in vivo and found to inhibit calcineurin-dependent signaling in T cells. One of these compounds was also found to cause elevated basal expression of a TGF $\beta$-R/SMAD-dependent reporter gene. These results demonstrate the capability of the RTA small molecule screening assay for discovery of potentially novel therapeutic compounds.
\end{abstract}

\section{INTRODUCTION}

Most biochemical processes are mediated through specific interactions between proteins, and a primary goal of functional genomics is to elucidate complete protein interaction networks. One consequence of this focus is the potential for development of protein interaction antagonists that could target highly specific biochemical pathways. The diversity of small molecule protein interaction inhibitors that have been described has expanded considerably over the past several years $(1,2)$, lending support to the feasibility of pursuing this strategy for drug discovery. Consequently, for this purpose, a variety of in vitro biochemical $(3,4)$, as well as bacterial (5), yeast (6-8), and mammalian cell $(9,10)$ based genetic strategies have been designed to enable high-throughput screening of small molecule compound libraries for protein interaction inhibitors. The majority of yeast-based strategies are derived from variations of the two-hybrid and inter- action trap systems in which interaction between DNA binding domain bait and activation domain prey fusions causes activation of a reporter gene $(6,7)$. Conversely, disruption of the bait-prey fusion interaction by an antagonist prevents recruitment of the activation domain prey and thereby causes loss of reporter gene expression. One inherent difficulty when using these systems for screening small molecule libraries is that hits representing potential interaction inhibitors produce a negative result (loss of signal), which need to be distinguished from effects due to toxicity or nonspecific inhibition of gene activation.

We have previously described a modified yeast two-hybrid strategy for use with transcriptional activator bait proteins, designated the repressed transactivator (RTA) system (11). Proteins that activate transcription typically cannot be used as bait fusions in the two-hybrid system, because they cause reporter gene expression in the absence of an interacting prey fusion protein.
In the RTA system, the bait fusion protein must be capable of activating transcription, while the prey proteins are fused to the $\mathrm{N}$-terminal transcriptional repression domain (RD) of Tup1, a general repressor of the budding yeast Saccharomyces (see Figure 1). In the absence of an interacting prey, activation of a URA3 reporter gene by the bait renders yeast sensitive to 5-fluoroorotic acid (5-FOA), which is converted to a toxic metabolite in a pathway dependent upon orotidine- $5^{\prime}$ phosphate (OMP) decarboxylase, the catalytic product of URA3 (see Figure 1A). Interaction between the activator bait and a Tup1 RD prey fusion causes repression of the URA3 reporter gene, thereby enabling growth on 5-FOA (see Figure 1B). This strategy has been used to identify novel interactions with a variety of transcriptional activators, including herpes simplex virus 1 (HSV1) regulatory protein VP16 (11), c-myc (12), and the androgen receptor (13).

Because interaction between the bait and prey fusions causes repression of 


\section{Research Reports}

A

B

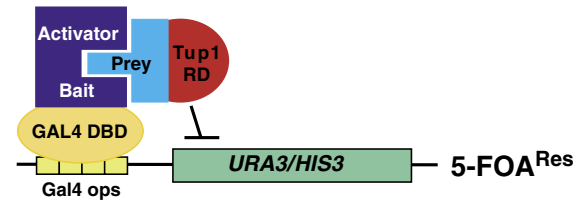

C

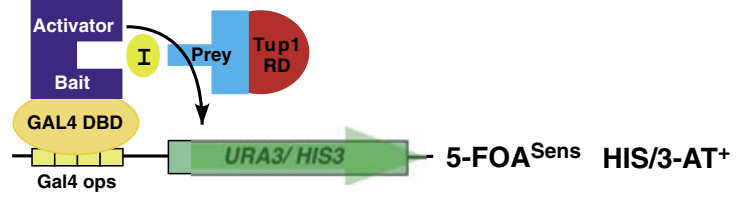

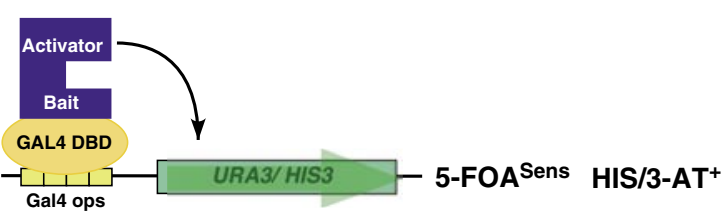

Figure 1. Representation of the repressed transactivator (RTA) system and its use for identification of protein interaction inhibitors. (A) Transcriptional activator bait proteins fused to the GAL4 DNA binding domain (GAL4 DBD) cause activation of $U R A 3$ and HIS3 reporter genes causing sensitivity to 5-fluoroorotic acid (5-FOA) and growth on 3-aminotriazole (3-AT), respectively. (B) Interaction of a Tup1 repression domain (RD) prey fusion with the bait causes repression of reporter gene expression, enabling growth on 5FOA and preventing growth on 3-AT. (C) Inhibitors of the baitprey interaction cause reporter gene derepression and growth on 3-AT.

reporter gene expression, we reasoned that the RTA system would be suited for identifying protein interaction inhibitors. Disruption of the interaction would cause reactivation of reporter genes, thereby producing a positive readout for potential hits in small molecule screens (see Figure 1C). In this report, we describe modifications that facilitate identification of protein interaction inhibitors and demonstrate feasibility of the strategy using several well-defined protein interactions and previously characterized inhibitors. To demonstrate utility, we adapted the system for high-throughput screening of small molecule compound libraries and have identified novel potential interaction inhibitors for four independent protein interactions. Compounds discovered that inhibit interaction between FKBP12 and the transforming growth factor $\beta$ receptor (TGF $\beta-R) C$ terminus were shown to inhibit signaling in vivo, demonstrating effectiveness of this system for identifying potential novel lead pharmacological agents.

\section{MATERIALS AND METHODS}

\section{Yeast Strains and Plasmids}

The bait plasmids expressing GAL4 DNA binding domain fusions from an $A R S-C E N-T R P I$ backbone are derived from pY1 (14). The activated pYV bait plasmids were derived from pY1 by inserting GAL4 DNA binding domain and VP16 encoding NcoI/BglII and $B g l \mathrm{II} / E c o$ RI PCR fragments, respectively. The multicopy LEU2 vector expressing Tup1 RD from the MET3 promoter (pIOX008) was produced by inserting an $A p a \mathrm{I} / S p h \mathrm{I}$ fragment (made blunt) from pBD1 (11) into YEpLac181 (15) cut with $P v u I I / N a r I$ (also made blunt). Plasmid pIOX274 (LEU2, ARS-CEN prey) was produced by inserting a PGK1 promoter fragment amplified from yeast genomic DNA and a Tup1 RD-multiple cloning site (MCS)-ADH1 terminator fragment from $\mathrm{pBD} 1$ into the $P v u \mathrm{II} /$ DraIII sites of pRS315 (16). The GAL4-VP16TGF $\beta-R$ C terminus (GV-TGF $\beta-R)$ and GAL4-VP16-thyroid hormone receptor $\alpha(\mathrm{GV}-\mathrm{TR} \alpha)$ bait and FKBP12 and nuclear receptor co-repressor 1 (Ncor1) prey plasmids contain inserts produced by amplification from cDNA clones in pYV1 and pIOX008 or pIOX274. The ATF4 and Nrf2 activator bait plasmids were recovered from an activator bait $\mathrm{T}$ cell cDNA library screen (K. Lund et al., unpublished data) and the $\mathrm{p} 300 /$ CBP Tup1 prey fusion plasmid from RTA interactions screens using these constructs as bait fusions.

The LG-HIS3 reporter plasmid (pIOX179) was derived from the ade8 disintegrator pIS112 (17) and contains a LEU3 operator and 10 concatamerized GAL4 binding sites upstream of a HIS3 open reading frame (ORF)-ADHI terminator fragment. Yeast strain YIOX001 (MATo, ade2, his3, leu2, trp1, ura3, can1, gal4, ade8::LG-HIS3) was produced by disintegration of ade 8 in strain YJR10 (18) with pIOX179. Strain YIOX124 (MATo, ade2, his3, leu2, trp1, ura3, can1, gal4, cyhr, ade $:: L G-H I S 3)$ was produced from a cross between YIOX001 and YIOX123 (MATo, ura3, leu2, his3, ade2, lys2, trpl, cyhr).

\section{Compound Libraries and Reagents}

Ascomycin, phorbol 12-myristate 13-acetate (PMA), ionomycin, and 3aminotriazole (3-AT) were all obtained from Sigma-Aldrich, (St. Louis, MO, USA). Cycloheximide, FK506, and TGF $\beta$ were obtained from EMD Chemicals (Calbiochem ${ }^{\circledR}$; La Jolla, CA, USA). The Genesis Plus small compound library, consisting of 960 biologically active compounds, was obtained from MicroSource Discovery Systems (Gaylordsville, CT, USA) supplied in 12-deep-well microtiter plates at $10 \mathrm{mM}$ concentration dissolved in dimethyl sulfoxide (DMSO). The 23,247-compound library was obtained from Chemical Diversity Labs (San Diego, CA, USA) and was provided as $0.2-\mu \mathrm{mol}$ aliquots in $1 \mathrm{mM}$ stocks (in DMSO) contained in 96-well plates. For screening purposes, we aliquoted the library into 108 plates containing 88 compounds and 174 plates containing 80 compounds.

\section{Small Molecule Inhibitor Assay}

Yeast strain YIOX001, transformed with the appropriate bait and prey plasmids, were grown in synthetic dextrose (SD) medium lacking tryptophan and leucine (SD-TL) to an absorbance $\mathrm{A}_{600 \mathrm{~nm}}$ approximately 1.0 and then diluted to $\mathrm{A}_{600 \mathrm{~nm}}$ of 0.02 into SD medium lacking tryptophan, leucine, histidine, and methionine (SD-TLHM). Fifty microliters diluted culture were aliquoted into 96-well flat bottomed microtiter plates containing $50 \mu \mathrm{L}$ SD-TLHM (containing 50 $\mathrm{mM} 2 \times 3$-AT). Five microliters each compound (diluted in SD-TLHM plus 3-AT with $1.5 \%$ DMSO) were added to the wells at a final concentration of $5 \mu \mathrm{M}$, and the microtiter plates were 


\section{Research Reports}

incubated at $30^{\circ} \mathrm{C}$ for $48 \mathrm{~h}$ in humidity chambers. Each plate contained control wells with yeast cotransformed with the bait and prey plasmids but with no added compound (DMSO control), yeast cotransformed with the bait and the Tup1 RD expression vector, and wells with $100 \mu \mathrm{L}$ SD-TLHM plus 3-AT media for use as an absorbance blank. Following growth, the yeast were resuspended using a multichannel pipetor, and absorbance $\mathrm{A}_{600 \mathrm{~nm}}$ was determined using a microplate reader (SpectraMax ${ }^{\circledR}$ Plus384; Molecular Devices, Sunnyvale, CA, USA). All assays were performed in triplicate, using a Biomek ${ }^{\circledR}$ FX liquid handler (Beckman Coulter, Mississauga, ON, Canada) equipped with a 96-channel head as outlined in the supplementary material available online at www. BioTechniques.com. The percent inhibition was calculated for each well as:

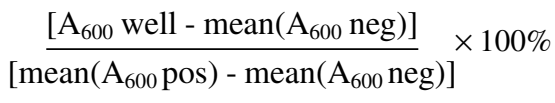

where $\mathrm{A}_{600}$ well equals absorbance of each well containing a compound, $\mathrm{A}_{600}$ neg equals absorbance of the negative control wells with yeast expressing the bait and prey without added compound, and $\mathrm{A}_{600}$ pos equals absorbance of wells with yeast co-expressing the bait and unfused Tup1 RD prey.

\section{Mammalian Cell Assays}

Jurkat and Jurkat W1 cells bearing the integrated human immunodeficiency virus type 1 (HIV-1) long terminal repeat (LTR) luciferase reporter (19) were grown in RPMI 1640 containing $10 \%$ fetal bovine serum (FBS) and treated with the IOX compounds at the indicated concentration for $2 \mathrm{~h}$ prior to stimulation with $10 \mathrm{ng} / \mathrm{mL}$ PMA and $1 \mu \mathrm{M}$ ionomycin. Twenty-four hours later, the cells were collected and the culture supernatant was assayed for interleukin 2 (IL-2) by enzyme-linked immunosorbent assay (ELISA) (20), or the cell pellets were washed once with phosphate-buffered saline (PBS) and lysed in luciferase lysis buffer (Promega, Madison, WI, USA). Fifty microliters lysate were incubated with an equal volume of luciferase substrate, and reactions were read using a microplate luminometer (The Reporter ${ }^{\circledR}$; Turner Designs, Sunnyvale, CA, USA) as described previously (19). To measure TGF $\beta$ response, Mv1Lu mink lung epithelial cells were cotransfected with $0.5 \mu \mathrm{g}$ SMAD-response reporter PAI 3TP-Lux (21) and $0.05 \mu \mathrm{g}$ PGK $\beta$-galactosidase ( $\beta$-gal) construct per well in $2 \times 3$ well plates. Twenty-four hours posttransfection, the cells were treated with the indicated concentration of IOX compound for $2 \mathrm{~h}$, prior to the addition of $0.25 \mathrm{nM}$ TGF $\beta$. The cells were incubated an additional $24 \mathrm{~h}$ prior to harvesting and assaying luciferase activity as described here.

\section{RESULTS}

\section{Small Molecule Assay Development}

The RTA yeast two-hybrid system was designed to identify novel interactions with transcription factors and, to date, has been used for screening cDNA libraries to identify novel interactions with several different transcriptional activator bait proteins (11-13). In the initial version of this system (11), GAL4 DNA binding domain baits are expressed from a single-copy (ARS-CEN) plasmid vector with a TRP1 marker, while the Tup1 $\mathrm{RD}$ prey fusions are produced from a multicopy $(2-\mu \mathrm{m})$ vector with a HIS3 marker. Interaction between bait and prey Tup1 RD prey fusions is detected using a URA3 reporter gene bearing upstream GAL4 operators; repression of the reporter caused by bait and prey interaction produces resistance to 5FOA (Figure 1, A and B).

To enable forward selection of protein interaction inhibitors within a multiwell plate growth assay, we developed a HIS3 reporter gene with a minimal promoter bearing upstream binding sites for GAL4 (see Figure 1 and Supplementary Figure S1A). HIS3 encodes imidazoleglycerol-phosphate dehydratase, which catalyzes the sixth step in histidine biosynthesis, an enzyme that is sensitive to competitive inhibition by 3-AT. Activation of the HIS3 reporter by the bait protein allows growth of his 3 null yeast on medium lacking histidine and containing 3-AT, and furthermore, elevated concentrations of 3-AT can be used to select for higher levels of HIS3 expression (22). Consequently, the relative strength of transcriptional activation by a particular bait fusion, on its own or caused by disruption of an interaction with a Tup1 RD prey fusion (Figure 1C), can be assessed by monitoring the growth rate of yeast in medium lacking histidine and containing appropriate concentrations of 3-AT. To accommodate use of the HIS3 reporter, we also constructed a series of Tup1 RD prey plasmids bearing $L E U 2$ as a selectable marker (Supplementary Figure S1, B and C).

Two additional modifications were introduced to allow the use of any protein as bait, whether capable of activating transcription or not. First, we introduced operators for the transcription factor LEU3 upstream of the GAL4 binding sites in the promoter for the HIS3 reporter gene (hereafter designated the LG-HIS3 reporter) (see Figure S1A) to produce modest constitutive expression. In combination with even weak GAL4 DNA binding domain activator fusions, expression of this reporter can be detected on his3-AT medium (Figure 2A and data not shown). Secondly, we created a set of vectors to simplify generation of fusions with GAL4-VP16 (Supplementary Figure S1D). The C-terminal domain of HSV-1 VP16 is a well-characterized, highly efficient transactivation domain (11) and is commonly used for in vitro transcription studies and to regulate gene expression as DNA binding domain fusions in a variety of eukaryotic species. We find that most proteins that are incapable of transcriptional activation on their own can be fused with GAL4-VP16 to create a tripartite protein capable of activating transcription at a level comparable to GAL4-VP16 itself (Figure 2A).

\section{Detection of Bait-Prey Interactions Using the HIS3 Reporter}

The combination of modifications described in the previous section were found to be effective for detecting interaction between a variety of proteins previously shown to associate in vitro and/or in vivo. To develop the system for high-throughput small 


\section{Research Reports}

molecule inhibitor screening, we used the well-defined interaction between the $\mathrm{C}$ terminus of the TGF $\beta-\mathrm{R}$ and the immunophilin FKBP12 (21). Neither of these proteins activate transcription on their own when fused to a DNA binding domain (data not shown), and therefore we created a GV-TGF $\beta$-R tripartite bait fusion. This fusion caused activation of the $L G$-HIS3 reporter gene as determined by growth of yeast on his- selective medium containing 3-AT (Figure 2A). Importantly, coexpression of the Tup1 RD fused to FKBP12 (Tup-FKBP12) prevented growth of yeast expressing the GVTGF $\beta$-R bait fusion, but not GAL4VP16, on his- 3-AT medium (Figure 2A), indicating repression of the HIS3 reporter gene produced by interaction between the TGF $\beta-R$ and FKBP12. The Tup1 RD produced on its own does not affect transcriptional activation by either bait (Figure 2A). This effect observed on agar plates could be reproduced by monitoring growth of yeast in 96-well plate liquid cultures containing his- 3-AT selective medium (Figure 2B). Yeast bearing the LG-HIS3 reporter and expressing the GV-TGF $\beta$ $\mathrm{R}$ fusion in combination with the Tup1 RD on its own grow efficiently in his- selective medium, as measured by absorbance $\mathrm{A}_{600 \mathrm{~nm}}$, and growth is impaired by approximately $50 \%$ when 25-45 mM 3-AT is included in the culture medium (Figure 2B, TFG $\beta$-RFKBP12). However, co-expression of Tup1-FKBP12 with the GV-TGF $\beta-R$ bait caused significant inhibition of growth in his- medium and completely prevented growth in the presence of 25 $\mathrm{mM}$ and higher concentrations of 3-AT (Figure 2B), demonstrating interaction between these fusions in the context of a multiwell plate growth assay.

We confirmed that the 3-AT growth assay was generally applicable for detecting protein-protein interactions using other previously characterized bait-prey combinations. The TR represses transcription by recruitment of Ncor1 (23). Because the TR $\alpha$ isoform does not activate transcription on its own as a fusion with GAL4 DNA binding domain in yeast (data not shown), we created a GV-TR $\alpha$ bait fusion, which activated transcription at a level comparable to GV-TGF $\beta-R$, as measured by

A
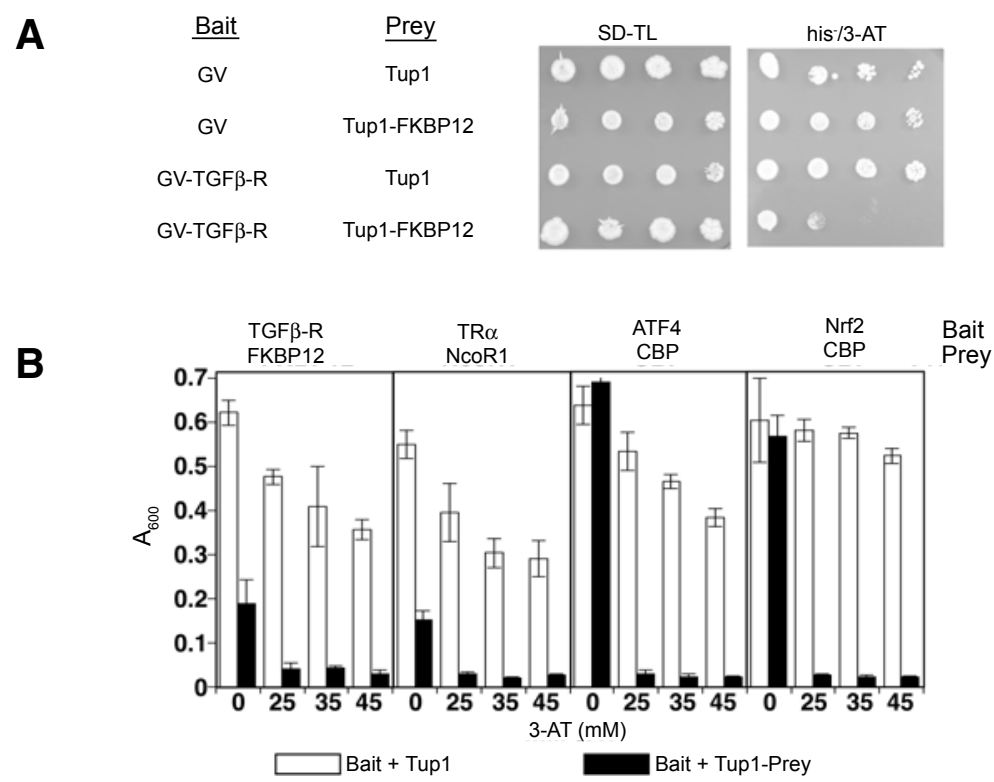

Figure 2. Interaction between activator bait fusions and Tup1 repression domain (RD) preys detected by growth on 3-aminotriazole (3-AT). (A) Yeast strain YIOX001 bearing the LG-HIS3 reporter gene and transformed with the bait-prey combinations indicated were spotted in 10-fold serial dilutions on synthetic dextrose (SD) agar plates lacking tryptophan and leucine (SD-TL) or SD agar plates lacking tryptophan, leucine, and methionine, but containing $25 \mathrm{mM} 3$-AT (his-/3-AT). The plates were incubated 4 days at $30^{\circ} \mathrm{C}$. (B) Yeast strain YIOX001 was cotransformed with plasmids expressing the GAL4-VP16transforming growth factor $\beta$ receptor $\mathrm{C}$ terminus (GV-TGF $\beta$-R)-Tup1-FKBP12, GAL4-VP16-thyroid hormone receptor $\alpha(\mathrm{GV}-\mathrm{TR} \alpha$ )-Tup1-nuclear receptor co-repressor 1 (Ncor1), GAL4-ATF4-Tup1-CBP, and GAL4-Nrf2-Tup1-CBP bait-prey fusions (closed bars, panels left to right, respectively) or the bait fusion plasmids cotransformed with the Tup1 RD vector (open bars). The transformants were diluted to an absorbance $\mathrm{A}_{600}$ of 0.01 and then grown for $48 \mathrm{~h}$ in selective medium containing the indicated concentration of 3-AT in 96-well plates.

growth in medium containing 3-AT (Figure 2B, TR $\alpha$ ). Co-expression of the Tup1 RD fused to Ncor1, but not the Tup1 RD produced on its own, inhibited growth of yeast expressing the GAL4VP16-TR $\alpha$ bait fusion (Figure 2B, $\operatorname{TR} \alpha$ ), indicating interaction between TR $\alpha$ and Ncor1. ATF4 and Nrf2 are two factors that cause strong transcriptional activation on their own when fused to the GAL4 DNA binding domain (Figure 2B, ATF4 and Nrf2). For both of these factors, we identified interactions with the histone acetyltransferase catalytic subunit p300/CBP in cDNA library screens, using RTA as previously described (data not shown). These interactions could be reproduced in vitro with recombinant proteins and are consistent with results suggesting that these factors functionally interact with CBP in vivo $(24,25)$. For both GAL4ATF4 and GAL4-Nrf2, co-expression of Tup1 RD fused to p300/CBP prevented

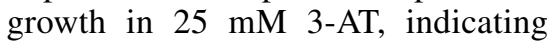
repression of the $L G$-HIS3 reporter gene due to interaction between the bait and prey fusions. In summary, these results demonstrate that the 3-AT growth assay in yeast bearing the $L G$-HIS3 reporter gene is effective for measuring interaction between a variety of different bait-prey partners.

\section{FKBP12 Ligands Inhibit Interaction with TGF $\beta$-R in the RTA System}

Interaction between TGF $\beta-\mathrm{R} C$ terminus and FKBP12 is disrupted by three known ligands of FKBP12FK506, ascomycin, and cycloheximide $(26,27)$. To validate the growth assay for identification of small molecule inhibitors, we examined the effect of these ligands on interaction between the GV-TGF $\beta-R$ bait and Tup1FKBP12 prey fusions, as measured by growth in 3-AT using 96-well plates. In the RTA system, the baits are expressed from a single-copy vector, whereas the Tup1 RD prey fusions are produced from a multicopy vector, and therefore the prey is generally overproduced relative to the bait fusion (11). 


\section{Research Reports}

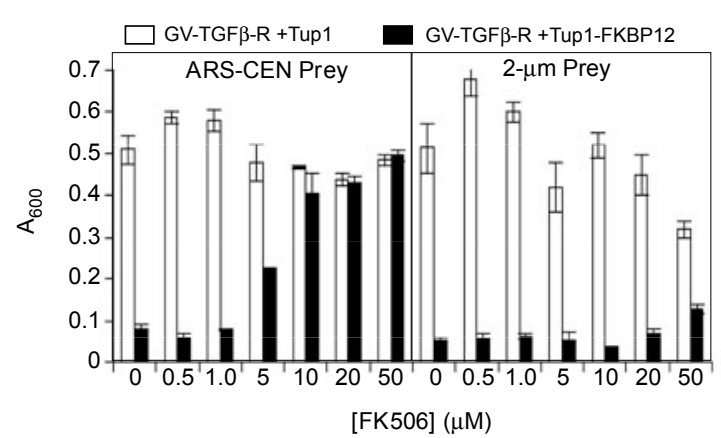

B

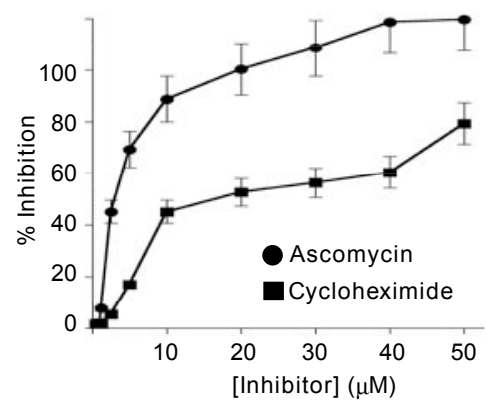

Figure 3. The FKBP12-transforming growth factor $\beta$ receptor (TGF $\beta$-R) repressessed transactivator (RTA) interaction is inhibited by FKBP12 ligands in yeast. (A) Yeast strain YIOX001 was cotransformed with the GAL4-VP16-TGF $\beta$-R $C$ terminus (GV-TGF $\beta$-R) bait plasmid and singlecopy (left panel) or multicopy (right panel) plasmids expressing Tup1FKBP12 (closed bars) or the Tup1 repression domain (RD) (open bars). The transformants were diluted to an absorbance $\mathrm{A}_{600}$ of 0.01 and then grown for $48 \mathrm{~h}$ in selective medium containing 25 $\mathrm{mM} 3$-aminotriazole (3-AT) and the indicated concentration of FK506 in 96-well plates. (B) The cycloheximideresistant yeast strain YIOX002 was cotransformed with the GV-TGF $\beta-R$ bait plasmid and the single-copy Tup1FKBP12 prey plasmid or the Tup1 vector control. Transformants were grown for $48 \mathrm{~h}$ in selective medium containing $25 \mathrm{mM} 3$-AT and the indicated concentration of ascomycin or cycloheximide in 96-well plates, and the absorbance measured at $\mathrm{A}_{600 \mathrm{~nm}}$. Shown is the percent inhibition of the FKBP12-TGF $\beta$-R interaction caused by the FKBP12 ligands calculated as described.

We expected that these differences in expression might affect sensitivity of the assay when screening for small molecule inhibitors or would produce a bias toward molecules that bound the bait rather than the prey fusion. To examine this possibility, we compared inhibition of the GV-TGF $\beta$-R-Tup1FKBP12 interaction by FK506 when the prey fusion was expressed from multicopy and single-copy vectors (Figure 3A). We found that expression of Tup1-FKBP12 from a multicopy vector rendered the interaction with GV-TGF $\beta$-R less sensitive to FK506 inhibition, causing poor grown in 3-AT medium (Figure 3A, right panel) than when the same fusion was produced from a single-copy vector (Figure 3A, left panel). These results indicated that greater sensitivity could be achieved by expressing both the bait and prey fusions from single-copy vectors.

FKPB12 binds a variety of ligands, and of these ascomycin and cycloheximide bind with vastly different affinities in vitro $(\mathrm{Kd}=1.0 \mathrm{nM}$ and $3.4 \mu \mathrm{M}$, respectively) $(26,27)$. We took advantage of this difference to examine the range of sensitivity we could

\section{Small Molecule Compound Library Screening Using RTA}

We initially developed a highthroughput robotic screening process using the 960 compound Genesis Plus collection of known biologically active compounds with the four protein interactions described in Figure 2: (i) GV-TGF $\beta$-R-FKBP12, (ii) ATF4p300/CBP, (iii) Nrf2-p300/CBP, and (iv) TR-Ncor1. A detailed summary of the process is diagrammed in Supplementary Figure S2A. Briefly, the reporter yeast strain transformed with the bait and prey plasmids were grown to mid-log phase, diluted to absorbance $\mathrm{A}_{600 \mathrm{~nm}}$ of 0.01 in selective medium containing $25 \mathrm{mM} 3$-AT, and dispensed into microtiter plates. The compound library samples were added to produce a final concentration of $5 \mu \mathrm{M}$. Each plate contained control wells with yeast expressing the bait and prey fusions but without any added compound (DMSO control), as well as wells containing yeast transformed with plasmids expressing the bait in combination with Tup1 RD as a positive control for growth (Supplementary Figure S2B). The plates were incubated at $30^{\circ} \mathrm{C}$ for $48 \mathrm{~h}$, and growth was measured by determination of absor-

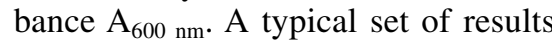
with the Genesis Plus collection using the GV-TGF $\beta$-R-FKBP12 interaction is shown in Supplementary Figure S3. Following the pilot studies, we scaled up the process to screen each of the four protein interactions with a diverse library of 23,247 compounds. In these screens, preliminary positive hits were defined as compounds that produced at least $50 \%$ inhibition of the bait-prey interaction with a standard deviation $<25 \%$ at a concentration of $5 \mu \mathrm{M}$. With these criteria, we identified a collection of specific and nonspecific inhibitors for each of the four bait-prey interactions (Table 1).

\section{Inhibitors of the FKB12-TGF $\beta$-R Interaction Affect Signaling In Vivo}

In activated $\mathrm{T}$ cells, FKBP12, regulates calcium mobilization to stimulate the nuclear factor of activated $\mathrm{T}$ cells (NFAT) transcription factors, which in combination with nuclear 


\section{Research Reports}

factor $\kappa B(N F-\kappa B)$ and activating protein 1 (AP1) causes induction of genes responsive to $\mathrm{T}$ cell receptor engagement (28). Additionally, FKBP12, through its interaction with the TGF $\beta-R \quad C$ terminus, inhibits receptor activation by $\operatorname{TGF} \beta$ (21). In our compound library screens, we identified six compounds that specifically inhibited the TGF $\beta-\mathrm{R} C$ terminus-FKBP12 interaction (Table 1). Upon retesting, five of these exhibited inhibition and specificity comparable to the initial screens, but only three were immediately available for resupply (Figure 4A). To further validate the compounds, we examined whether they affected FKBP12-dependent signaling by determining their effect on expression of NFAT and TGF $\beta$ responsive genes. IL-2 expression is induced in response to $\mathrm{T}$ cell receptor activation through parallel pathways that activate NFAT, AP1, and NF- $\kappa \mathrm{B}$, a response that can be mimicked by treatment with PMA and ionomycin (20). We found that all three compounds inhibited production of IL-2 in Jurkat T cells treated with PMA/ionomycin with a dose-dependent response, although only IOX13362 produced a response that plateaued at a concentration $<5$ $\mu \mathrm{M}$ (Figure 4B). For this compound, we calculated an inhibitory concentration of $50 \%\left(\mathrm{IC}_{50}\right)$ for inhibition of IL-2 production of $0.23 \mu \mathrm{M}$ (data not shown). To determine whether the inhibitory effect of these compounds on stimulation of IL-2 production reflected a general inhibitory effect on other FKBP12-dependent signaling, we also examined their effect on induction of the HIV-1 LTR. HIV-1 transcription is tightly coupled to $\mathrm{T}$ cell activation and, like the IL-2 promoter, is regulated by parallel pathways impinging on NFAT and NF- $\kappa \mathrm{B}$, as well as c-Ets/GA binding protein (GABP) $(29,30)$. For these experiments, we used a Jurkat $T$ cell line bearing a chromosomally integrated HIV-1 LTR-luciferase reporter gene. We found that each of the three compounds inhibited induction of the integrated LTR in response to PMA/ionomycin, which is consistent with an inhibitory effect on $\mathrm{T}$ cell signaling (Figure 4C). None of the three compounds had a detectable effect on cell viability at the concentrations tested as determined using an MTT cell proliferation assay (data not shown).

Response to TGF $\beta$ is affected primarily through regulation of the SMAD family transcription factors, which in unstimulated cells are bound to the unactivated TGF $\beta-R$ (31). Interaction of TGF $\beta$ with the receptor causes release of the SMADs, allowing their translocation to the nucleus for activation of responsive promoters (31), and FKBP12 negatively regulates signaling from the TGF $\beta$ receptor through its interaction with the cytoplasmic C-terminal domain $(21,32)$. Given this, we postulated that compounds that inhibit interaction between FKBP12 and the TGF $\beta$-R in the yeast assay might also affect response to TGF $\beta$ in vivo. To examine this possibility, we measured the effect of the compounds on expression of a SMADresponsive reporter gene. We found that two of the compounds, IOX14899 and IOX00250, had a slight inhibitory affect on TGF $\beta$-induced reporter gene expression at $5 \mu \mathrm{M}$ (Figure $4 \mathrm{D}$ ), a concentration that prevents induction of the HIV-1 LTR (Figure 4C), while the third compound, IOX13362, had no significant affect on induction of the reporter (Figure 4D). Interestingly, one of the compounds, IOX00250, caused

Table 1. Summary of Hits Identified in Small Molecule Screens

\begin{tabular}{|lccc|}
\hline Interaction & Total Hits $^{\mathbf{a}}$ & Specific Hits $^{\mathbf{b}}$ & Specific Hit Rate \\
\hline TGF $\beta$-RI/FKBP12 & 18 & 6 & $0.026 \%$ \\
TR $\alpha /$ Ncor1 & 97 & 86 & $0.370 \%$ \\
ATF4/CBP & 1 & 1 & $0.004 \%$ \\
Nrf2/CBP & 5 & 2 & $0.009 \%$ \\
aThe total number of compounds which caused at least 50\% inhibition of the indicated interaction, with a \\
standard error <25\%. \\
bThe number of compounds that specifically inhibited the indicated among the four screens performed \\
in parallel. \\
TGF $\beta-\mathrm{R}$, transforming growth factor $\beta$ receptor.
\end{tabular}

significantly elevated basal expression of the SMAD-responsive reporter in untreated cells (Figure 4D). This result suggests that one of the compounds selected as an FKBP12-TGF $\beta$-R interaction inhibitor may interfere with the inhibitory effect of FKBP12 in unstimulated cells to allow elevated basal signaling from the receptor. None of the three compounds had any affect on expression from the cotransfected PGK $\beta$-gal reporter construct (data not shown), which indicates that they specifically affect signaling to the IL-2 promoter, HIV-1 LTR, and the SMADresponsive reporter gene, rather than producing nonspecific effects on gene expression.

\section{DISCUSSION}

A major current focus of proteomics and functional genomics involves the delineation of complete protein interaction networks, and there is increasing interest in development of small molecule protein interaction inhibitors as potential therapies for targeting specific biological processes $(1,2,33)$. The feasibility of this approach for drug discovery comes with the realization that small molecule compounds can potentially disrupt specific interaction between proteins through a variety of mechanisms. Although protein interaction interfaces are relatively large, there is evidence that binding of small molecules to interaction hot spots, contacts that critically influence the affinity between two proteins, can effectively disable the protein interaction (2). Small molecule compounds could also indirectly inhibit protein interactions through binding allosteric sites and forcing conformational alterations that prohibit interaction with binding partners (2). Additionally, small molecules could potentially disrupt normal protein folding, thereby preventing formation of structures necessary for protein interactions or causing destabilization of either interacting partner (34). With these possibilities, it seems the bottleneck(s) in developing therapeutically viable small molecule protein interaction inhibitors are the lack of sufficiently diverse small molecule libraries targeted 


\section{Research Reports}

toward this purpose, in addition to an adequate variety of effective screening strategies.

In our assays, we screened four different protein interactions in parallel to enable the rigorous distinction of compounds that specifically inhibited a single protein interaction. Each of the four protein interactions produced a different hit profile. For example, the ATF4-CBP and Nrf2-CBP interactions produced very few hits, whereas the TR $\alpha /$ Ncor 1 interaction resulted in 86 specific hits (Table 1). These differences in hit rates between the various interactions might be influenced by the relative transcriptional activation strength of the bait as well as the affinity of the interacting prey partner. For example, the GV-TR $\alpha$ bait is a weaker activator than ATF4 or Nrf2. Therefore, the difference in the growth rate caused by the unrepressed GV-TR $\alpha$ bait relative to the rate caused by interaction with the Tup1-Ncor1 prey is smaller than the corresponding difference for the ATF4-CBP and Nrf2-CBP interactions (Figure 2). This smaller dynamic range might render this interaction more sensitive to inhibition by a wider range of compounds. Alternatively, the differences in hit rates could reflect an inherent sensitivity or resilience to inhibition of the interactions. For example, interaction of the TR with its ligands causes dissociation of Ncor1 (23), an effect we can observe using the RTA system (data not shown). Although we have not analyzed specific hits from the TR $\alpha /$ Ncor1 screen in detail, it is possible that some of these may mimic the effect of thyroid hormone. Conversely, the interaction of ATF4 and Nrf2 with p300/CBP may be inherently insensitive due to an intrinsically stronger interaction potential and/or multiple contact surfaces. Further characterization of these interactions will be necessary to enable predication of hit rates that could be expected for other protein interactions using the RTA small molecule screening assay.

We expected to observe compounds that produce hits with all four protein interactions, because in principle, inhibiting expression of the Tup1prey fusion or interference with the repressive effect of Tup1 should enable growth on 3-AT. The fact that we did not identify such compounds likely reflects the limited diversity of our library. However, we did observe 13 compounds that produced hits with two different interactions (Table 1 and data not shown). Understanding the mechanism(s) of action of such semispecific inhibitors will require further screens with additional diverse protein interactions. Importantly, we note that no previous study describing small molecule protein interaction inhibitor screens has used more than two interactions in parallel to determine specificity. In our studies, we did not observe any obvious structural similarities between the semi-specific compounds we observed in our screen, and it will be interesting to determine how frequently such compounds are observed in other types of small molecule inhibitor screens and with more diverse compound libraries. One possibility is that compounds that inhibit a pair of unrelated targets may do so because they bind similar structural motifs or are capable of intercalating between specific types of protein interactions.

The FKBP1-TGF $\beta-R$ interaction is well-characterized and has served as a model for validation of several previously described inhibitor screening strategies $(6,9,10)$. However, none of these previous studies used this interaction for screening compound libraries. FKBP12 regulates a combination of signaling mechanisms in activated T cells. Through its interaction with the IP3 receptor and intracellular calcium channels, FKBP12 regulates calcium release in response to $\mathrm{T}$ cell receptor stimulation (28). Calcium controls the activity of the phosphatase calcineurin, which dephosphorylates the transcription factor NFAT enabling its translocalization to the nucleus (37). NFAT in conjunction with FOXP3 activates IL-2 transcription (38) and, in combination with NF- $\kappa \mathrm{B}$, GABP, and RBF-2, the HIV-1 LTR in response to $\mathrm{T}$ cell receptor signaling $(29,30)$. The immunosupressive macrolide FK506 inhibits $\mathrm{T}$ cell signaling by binding FKBP12 to produce a complex that directly inhibits calcineurin (39). In addition to its role in lymphocyte activation, FKBP12, through its interaction with the TGF $\beta$-R, inhibits TGF $\beta$ mediated signaling $(21,40)$. FKBP12 binds to the unstimulated receptor and blocks activation by a mechanism that is thought to prevent leaky signaling (21). There is also evidence suggesting that FKBP12 may inhibit liganddependent TGF $\beta$-R internalization (41). Interaction of TGF $\beta-R$ and FKBP12 can be inhibited by FK506 (32) (Figure 2 ), and structural studies have shown that both FK506 and the TGFB-R C terminus interact with the same LeuPro binding pocket of FKBP12 $(42,43)$. Therefore, we reasoned that novel inhibitors of the TGF $\beta$-R-FKBP12 interaction would potentially inhibit calcineurin and NFAT-dependent signaling, and, consistent with this, we found that three inhibitors identified in our screen inhibited induction of IL2 and activation of the HIV-1 LTR in stimulated Jurkat T cells (Figure 4, B and $\mathrm{C}$ ).

One of the compounds (IOX00250) we identified as an FKBP12-TGF $\beta$ $\mathrm{R}$ interaction inhibitor also caused elevated transcription of a TGF $\beta /$ SMAD-dependent reporter gene in unstimulated cells (Figure 4D). A nonfunctional derivative of FK506 $(\mathrm{L} 685,818)$ was previously shown to cause elevated expression of the same reporter in response to low doses of TGF $\beta$ but had no effect in unstimulated cells (21), and it was suggested that FKBP12 may normally function to modulate the threshold response level of the receptor (40). Therefore, the effect of IOX00250 on basal signaling for SMAD-dependent reporter gene expression seems curious, given that L685,818 causes complete release of FKBP12 from the receptor without affecting basal signaling (40). FKBP12 is a member of the peptidyl-prolyl cis-transisomerase family, which are ubiquitously expressed and evolutionarily conserved (44). These proteins are thought to function as molecular chaperones that affect a variety of different cellular functions, and their prolyl isomerase activity and affinity for target proteins can be modulated or altered upon ligand binding (28). Furthermore, mammalian cells express seven different FKBPs with overlapping ligand specificity, which are found in distinct cytoplasmic and nuclear complexes with different target proteins (28). Consequently, the mechanism(s) 


\section{Research Reports}

A

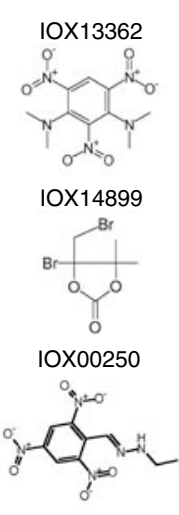

B

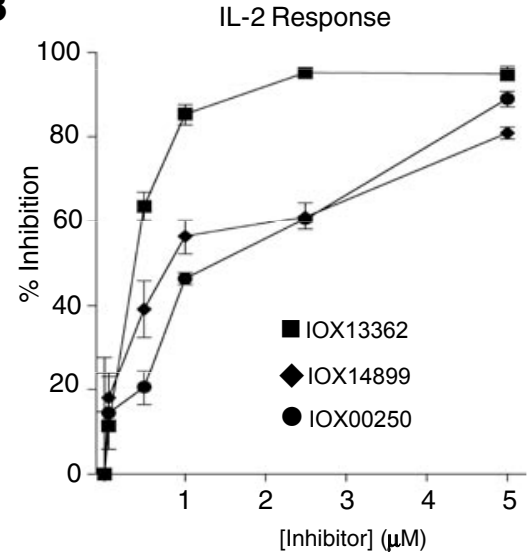

C

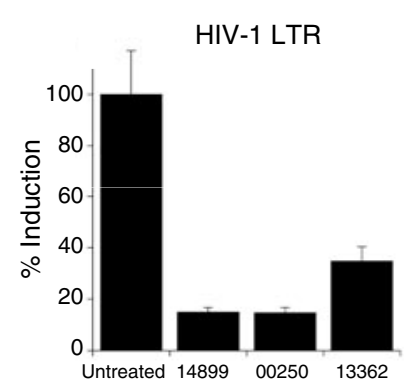

D
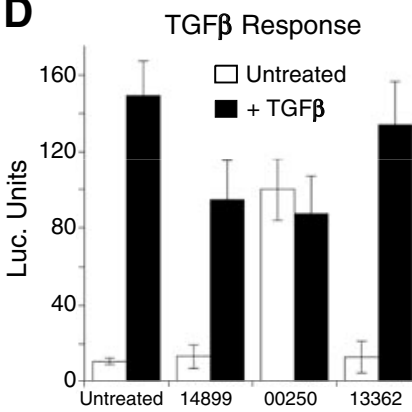

Figure 4. Small molecule inhibitors of the FKBP12-transforming growth factor $\beta$ receptor (TGF $\beta$ R) interaction affect calcineurin and TGF $\beta$-dependent signaling in vivo. (A) Structures of three compounds identified as specific inhibitors of the FKBP12-TGF $\beta-R$ interaction in repressed transactivator (RTA)-small molecule screens. (B) Jurkat T cells were treated with phorbol 12-myristate 13acetate (PMA)/ionomycin in the presence of the indicated concentration of FKBP12-TGF $\beta$-R inhibitor compound, and IL-2 production was measured by enzyme-linked immunosorbent assay (ELISA) 24 $\mathrm{h}$ poststimulation. Shown is the percent inhibition relative to untreated PMA/ionomycin-stimulated cells. (C) Jurkat W1 cells (19), bearing a chromosomally integrated human immunodeficiency virus type 1 (HIV-1) long terminal repeat (LTR)-luciferase reporter gene were stimulated with PMA/ionomycin in the absence (untreated) or presence of $5 \mu \mathrm{M}$ indicated FKBP12-TGF $\beta$-R inhibitor compound. Luciferase activity was measured $24 \mathrm{~h}$ poststimulation, and the activities were presented as a percentage of the untreated samples. (D) Mv1Lu cells were cotransfected with 0.5 $\mu \mathrm{g}$ SMAD-responsive PAI 3 TP Lux reporter plasmid (21) and $0.05 \mu \mathrm{g}$ pPGK- $\beta$-galactosidase ( $\beta$-gal). After $16 \mathrm{~h}$, the cells were treated with $5 \mu \mathrm{M}$ indicated FKBP12-TGF $\beta$-R inhibitor compound or were left untreated. Cells were then stimulated with $250 \mathrm{pM}$ TGF $\beta$ (solid bars) or left untreated (open bars) prior to determining relative luciferase activity.

by which IOX00250 inhibits calcineurin-dependent signaling but stimulates SMAD-dependent reporter gene activity will require verification that these effects specifically require FKBP12.

In conclusion, we describe a novel yeast-based two-hybrid strategy for screening small molecule inhibitors of protein interactions, and we have demonstrated its effectiveness in identifying potential inhibitors of four different interactions. The assay involves a simple and cost-effective forward growth assay that is easily adapted to high-throughput screening. This system adds to an increasingly diverse set of options for identifying small molecule protein interaction inhibitors $(4-7,9,10)$ necessary to meet the rapidly growing landscape of interaction networks $(2,33)$.

\section{ACKNOWLEDGMENTS}

Technical assistance was provided by Adrian Ally, Kane Tse, Limei Zhang, Jennifer Trinh, Georgia Tai, and Yu Yau. We thank Gabe Kalmar, Pauline Johnson, Roger Brent, and Michel Roberge for useful discussions. The PAI 3TP-Lux reporter plasmid was a generous gift from Joan Massague. This re- search was supported by funds from the Canadian Institutes of Health Research operating grant MOP-77807.

\section{COMPETING INTERESTS STATEMENT}

M.H. and I.S. are shareholders of Interomex Biopharmaceuticals, Inc., which holds intellectual property rights to the RTA system. The other authors declare no competing interests.

\section{REFERENCES}

1.Pagliaro, L., J. Felding, K. Audouze, S.J. Nielsen, R.B. Terry, C. Krog-Jensen, and S. Butcher. 2004. Emerging classes of proteinprotein interaction inhibitors and new tools for their development. Curr. Opin. Chem. Biol. 8:442-449.

2. Arkin, M.R. and J.A. Wells. 2004. Smallmolecule inhibitors of protein-protein interactions: progressing towards the dream. Nat. Rev. Drug Discov. 3:301-317.

3. Bergendahl, V., T. Heyduk, and R.R. Burgess. 2003. Luminescence resonance energy transfer-based high-throughput screening assay for inhibitors of essential protein-protein interactions in bacterial RNA polymerase Appl. Environ. Microbiol. 69:1492-1498.

4. Best, J.L., C.A. Amezcua, B. Mayr, L. Flechner, C.M. Murawsky, B. Emerson, T. Zor, K.H. Gardner, and M. Montminy. 2004. Identification of small-molecule antagonists that inhibit an activator: coactivator interaction. Proc. Natl. Acad. Sci. USA 101:17622-17627.

5.Horswill, A.R., S.N. Savinov, and S.J. Benkovic. 2004. A systematic method for identifying small-molecule modulators of protein-protein interactions. Proc. Natl. Acad. Sci. USA 101:15591-15596.

6. Huang, J. and S.L. Schreiber. 1997. A yeast genetic system for selecting small molecule inhibitors of protein-protein interactions in nanodroplets. Proc. Natl. Acad. Sci. USA 94:13396-13401.

7. Kato-Stankiewicz, J., I. Hakimi, G. Zhi, J. Zhang, I. Serebriiskii, L. Guo, H. Edamatsu, H. Koide, et al. 2002. Inhibitors of Ras/Raf-1 interaction identified by twohybrid screening revert Ras-dependent transformation phenotypes in human cancer cells. Proc. Natl. Acad. Sci. USA 99:14398-14403.

8. Vidal, M., R.K. Brachmann, A. Fattaey, E. Harlow, and J.D. Boeke. 1996. Reverse two-hybrid and one-hybrid systems to detect dissociation of protein-protein and DNA-protein interactions. Proc. Natl. Acad. Sci. USA 93:10315-10320.

9. Eyckerman, S., I. Lemmens, D. Catteeuw, A. Verhee, J. Vandekerckhove, S. Lievens, and J. Tavernier. 2005. Reverse MAPPIT: screening for protein-protein interaction modifiers in mammalian cells. Nat. Methods 2:427-433

10.Zhao, H.F., T. Kiyota, S. Chowdhury, E. Purisima, D. Banville, Y. Konishi, and S.H. 


\section{Research Reports}

Shen. 2004. A mammalian genetic system to screen for small molecules capable of disrupting protein-protein interactions. Anal. Chem. 76:2922-2927.

11. Hirst, M., C. Ho, L. Sabourin, M. Rudnicki, L. Penn, and I. Sadowski. 2001. A two-hybrid system for transactivator bait proteins. Proc. Natl. Acad. Sci. USA 98:8726-8731.

12. Wafa, L.A., H. Cheng, M.A. Rao, C.C. Nelson, M. Cox, M. Hirst, I. Sadowski, and P.S. Rennie. 2003. Isolation and identification of L-dopa decarboxylase as a protein that binds to and enhances transcriptional activity of the androgen receptor using the repressed transactivator yeast two-hybrid system. Biochem. J. 375:373-383.

13. Huang, A., C.S. Ho, R. Ponzielli, D. Barsyte-Lovejoy, E. Bouffet, D. Picard, C.E. Hawkins, and L.Z. Penn. 2005. Identification of a novel c-Myc protein interactor, JPO2, with transforming activity in medulloblastoma cells. Cancer Res. 65:56075619.

14. Sadowski, I., B. Bell, P. Broad, and M. Hollis. 1992. GAL4 fusion vectors for expression in yeast or mammalian cells. Gene 118:137-141.

15. Gietz, R.D. and A. Sugino. 1988. New yeastEscherichia coli shuttle vectors constructed with in vitro mutagenized yeast genes lacking six-base pair restriction sites. Gene 74:527534.

16. Sikorski, R.S. and P. Hieter. 1989. A system of shuttle vectors and yeast host strains designed for efficient manipulation of DNA in Saccharomyces cerevisiae. Genetics 122:1927.

17. Sadowski, I., T.C. Su, and J. Parent. 2007. Disintegrator vectors for single copy yeast chromosomal integration. Yeast Feb 22;[Epub ahead of print]

18. Rohde, J.R., J. Trinh, and I. Sadowski. 2000. Multiple signals regulate GAL transcription in yeast. Mol. Cell. Biol. 20:38803886.

19. Chen, J., T. Malcolm, M.C. Estable, R.G. Roeder, and I. Sadowski. 2005. TFII-I regulates induction of chromosomally integrated human immunodeficiency virus type 1 long terminal repeat in cooperation with USF. J. Virol. 79:4396-4406.

20. Monner, D.A., K.F. Bierend, and P.F. Muhlradt. 1986. Induction of lymphokine synthesis in peripheral blood mononuclear cells with phorbol ester and calcium ionophore allows precise measurement of individual variations in capacity to produce IL 2 . Lymphokine Res. 5(Suppl 1):S67-S73.

21. Wang, T., B.Y. Li, P.D. Danielson, P.C. Shah, S. Rockwell, R.J. Lechleider, J. Martin, T. Manganaro, and P.K. Donahoe. 1996. The immunophilin FKBP12 functions as a common inhibitor of the TGF beta family type I receptors. Cell 86:435-444.

22. Bartel, P.L. and S. Fields. 1995. Analyzing protein-protein interactions using two-hybrid system. Methods Enzymol. 254:241-263.

23. Makowski, A., S. Brzostek, R.N. Cohen, and A.N. Hollenberg. 2003. Determination of nuclear receptor corepressor interactions with the thyroid hormone receptor. Mol. Endocrinol. 17:273-286.

24. Liang, G. and T. Hai. 1997. Characterization of human activating transcription factor 4 , a transcriptional activator that interacts with multiple domains of cAMP-responsive element-binding protein (CREB)-binding protein. J. Biol. Chem. 272:24088-24095.

25. Katoh, Y., K. Itoh, E. Yoshida, M. Miyagishi, A. Fukamizu, and $M$. Yamamoto. 2001. Two domains of Nrf2 cooperatively bind CBP, a CREB binding protein, and synergistically activate transcription. Genes Cells 6:857-868.

26. Christner, C., R. Wyrwa, S. Marsch, G. Kullertz, R. Thiericke, S. Grabley, D. Schumann, and G. Fischer. 1999. Synthesis and cytotoxic evaluation of cycloheximide derivatives as potential inhibitors of FKBP12 with neuroregenerative properties. J. Med. Chem. 42:3615-3622.

27. Kawai, M., B.C. Lane, G.C. Hsieh, K.W. Mollison, G.W. Carter, and J.R. Luly. 1993. Structure-activity profiles of macrolactam immunosuppressant FK-506 analogues. FEBS Lett. 316:107-113.

28. Dumont, F.J. 2000. FK506, an immunosuppressant targeting calcineurin function. Curr. Med. Chem. 7:731-748.

29. Brooks, D.G., P.A. Arlen, L. Gao, C.M. Kitchen, and J.A. Zack. 2003. Identification of T cell-signaling pathways that stimulate latent HIV in primary cells. Proc. Natl. Acad. Sci. USA 100:12955-12960.

30. Sadowski, I. and D.A. Mitchell. 2005. TFIII and USF (RBF-2) regulate Ras/MAPK-responsive HIV-1 transcription in T cells. Eur. J. Cancer 41:2528-2536.

31. Shi, Y. and J. Massague. 2003. Mechanisms of TGF-beta signaling from cell membrane to the nucleus. Cell 113:685-700.

32. Wang, T., P.K. Donahoe, and A.S. Zervos. 1994. Specific interaction of type I receptors of the TGF-beta family with the immunophilin FKBP-12. Science 265:674-676.

33. Chaudhuri, A. and J. Chant. 2005. Proteininteraction mapping in search of effective drug targets. Bioessays 27:958-969.

34.Archakov, A.I., V.M. Govorun, A.V. Dubanov, Y.D. Ivanov, A.V. Veselovsky, P. Lewi, and P. Janssen. 2003. Protein-protein interactions as a target for drugs in proteomics. Proteomics 3:380-391.

35. Kornberg, R.D. 2005. Mediator and the mechanism of transcriptional activation. Trends Biochem. Sci. 30:235-239.

36. Lee, T.I. and R.A. Young. 2000. Transcription of eukaryotic protein-coding genes. Annu. Rev. Genet. 34:77-137.

37. Li, T.K., S. Baksh, A.D. Cristillo, and B.E. Bierer. 2002. Calcium- and FK506-independent interaction between the immunophilin FKBP51 and calcineurin. J. Cell. Biochem. 84:460-471.

38. Wu, Y., M. Borde, V. Heissmeyer, M. Feuerer, A.D. Lapan, J.C. Stroud, D.L. Bates, L. Guo, et al. 2006. FOXP3 controls regulatory $\mathrm{T}$ cell function through cooperation with NFAT. Cell 126:375-387.

39. Liu, J., J.D. Farmer, Jr., W.S. Lane, J. Friedman, I. Weissman, and S.L. Schreiber. 1991. Calcineurin is a common target of cyclophilin-cyclosporin A and FKBP-FK506 complexes. Cell 66:807-815.

40. Wang, T. and P.K. Donahoe. 2004. The immunophilin FKBP12: a molecular guardian of the TGF-beta family type I receptors. Front. Biosci. 9:619-631.

41. Yao, D., J.J. Dore, Jr., and E.B. Leof. 2000. FKBP12 is a negative regulator of transform- ing growth factor-beta receptor internalization. J. Biol. Chem. 275:13149-13154.

42. Huse, M., Y.G. Chen, J. Massague, and J. Kuriyan. 1999. Crystal structure of the cytoplasmic domain of the type I TGF beta receptor in complex with FKBP12. Cell 96:425-436.

43. Chen, Y.G., F. Liu, and J. Massague. 1997. Mechanism of TGFbeta receptor inhibition by FKBP12. EMBO J. 16:3866-3876.

44. Schiene-Fischer, C. and C. Yu. 2001 Receptor accessory folding helper enzymes: the functional role of peptidyl prolyl cis/trans isomerases. FEBS Lett. 495:1-6.

Received 17 November 2006; accepted 9 January 2007.

Address correspondence to Ivan Sadowski, Department of Biochemistry and Molecular Biology, University of British Columbia, 2130 Health Sciences Mall, Vancouver, BC, V6T 1Z3, Canada. e-mail: sadowski@interchange.ubc.ca

To purchase reprints of this article, contact: Reprints@BioTechniques.com 